\title{
Video gaming in school children- how much is enough?
}

\author{
(1)

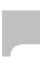 \\ Jesus Pujol, MD ${ }^{1,2}$; Raquel Fenoll, $\mathrm{MSc}^{1}$; Joan Forns, $\mathrm{PhD}^{3-5}$; Ben J. Harrison, $\mathrm{PhD}^{6}$; Gerard \\ Martínez-Vilavella, $\mathrm{MSc}^{1}$; Dídac Macià, $\mathrm{MSc}^{1}$; Mar Alvarez-Pedrerol, $\mathrm{PhD}^{3-5}$; Laura Blanco- \\ Hinojo, $\mathrm{PhD}^{1}$; Sofía González-Ortiz, $\mathrm{MD}^{7}$; Joan Deus, $\mathrm{PhD}^{1,8,9}$; Jordi Sunyer, $\mathrm{MD}^{3-5,10}$ \\ 2 \\ ${ }^{1}$ MRI Research Unit, Department of Radiology, Hospital del Mar, Barcelona, Spain \\ ${ }^{2}$ Centro Investigación Biomédica en Red de Salud Mental, CIBERSAM G21, Barcelona, Spain \\ ${ }^{3}$ Centre for Research in Environmental Epidemiology (CREAL), Barcelona, Catalonia, Spain \\ ${ }^{4}$ Pompeu Fabra University, Barcelona, Catalonia, Spain \\ ${ }^{5}$ Ciber on Epidemiology and Public Health (CIBERESP), Spain. \\ ${ }^{6}$ Melbourne Neuropsychiatry Centre, Department of Psychiatry, The University of Melbourne, \\ Melbourne, Australia. \\ ${ }^{7}$ Department of Radiology, Hospital del Mar, Barcelona, Spain \\ ${ }^{8}$ Guttmann Neurorehabilitation Institute, Autonomous University of Barcelona, Spain \\ ${ }^{9}$ Department of Clinical and Health Psychology, Autonomous University of Barcelona, Spain \\ ${ }^{10}$ IMIM (Hospital del Mar Medical Research Institute), Barcelona, Catalonia, Spain
}

Running head: Video gaming- how much is enough?

Number of characters in the title; 52 and running head; 33.

Number of words in the abstract, 247, Introduction; 212, Discussion 860, and the body of the manuscript; 3,846 .

Number of figures; 5, (color figures; 3), and tables; 2 .

Supplementary Tables; 1.

Corresponding author: Dr. Jesus Pujol. MRI Department, Hospital del Mar, Passeig Marítim 25-29. 08003, Barcelona, Spain. Email: 21404jpn@comb.cat Telephone: +34932212180 Fax: +34932212181

This is the author manuscript accepted for publication and has undergone full peer review but has not been through the copyediting, typesetting, pagination and proofreading process, which may lead to differences between this version and the Version record. Please cite this article as doi:10.1002/ ana.24745. 


\section{Abstract}

Objective: Despite extensive debate, the proposed benefits and risks of video gaming in young people remain to be empirically clarified, particularly in regards an optimal level of use. Methods: In 2,442 children aged 7-11 years, we have investigated relationships between weekly video game use, selected cognitive abilities and conduct-related problems. A large subgroup of these children $(\mathrm{N}=260)$ was further examined with MRI approximately one year later to assess the impact of video gaming on brain structure and function. Results: Playing video games for 1 hour per week was associated with faster and more consistent psychomotor responses to visual stimulation. Remarkably, no further change in motor speed was identified in children playing more than 2 hours a week. By comparison, the weekly time spent gaming was steadily associated with conduct problems, peer conflicts and reduced prosocial abilities. These negative implications were clearly visible only in children at the extreme of our game playing distribution with 9 hours or more of video gaming per week. At a neural level, changes associated with gaming were most evident in basal ganglia white matter and functional connectivity. Interpretation: Significantly better visuo-motor skills can be seen in school children playing video games, even with relatively small amounts of use. Frequent weekly use, by contrast, was associated with conduct problems. Further studies are needed to determine whether moderate video gaming causes improved visuo-motor skills and whether excessive video gaming causes conduct problems, or whether children who already have these characteristics simply play more video games. 


\section{Introduction}

The pros and cons of video gaming in children remain openly debated ${ }^{1,2}$. While there is some evidence to suggest that video gaming can improve particular cognitive abilities in youth ${ }^{3,4}$, other evidence links it with conduct-related problems and increased risk towards disorders of addiction $^{5,6}$. One possibility is that video gaming per se is neither good nor bad, but its level of use makes it so, which begs the question, how much is enough?

We performed the current study in 2,442 school-age children to investigate relationships between average weekly video game use and both cognitive performance and conduct-related problems. Considering video gaming as a training exercise based on repetitive use and on the basis of previous research ${ }^{3}$, we predicted that video gaming in school children would have a principal beneficial effect on speed of mental processing (e.g., reaction time and chronometric measurements of attention) and a marginal influence on more innate cognitive capabilities (e.g., working memory ${ }^{7,8}$ ).

A sub-group of this population $(n=260)$ was further examined with MRI approximately one year later to assess the impact of video gaming on brain structure and function. In the brain, video gaming could more notably reinforce neural connections, with a major effect presumably on frontal-basal ganglia circuits, which are central for the acquisition of new skills through practice ${ }^{9-11}$. 


\section{Methods}

\section{Participants}

This study was developed in the context of a large-scale project designed to assess the effects of environmental factors on brain development in children (BREATHE, The European Commission: FP7-ERC-2010-AdG, ID 268479). Study design and participant selection have been described in full detail elsewhere ${ }^{12,13}$. In short, the BREATHE project recruited 2,897 children from 39 schools to form a representative sample of children in Barcelona aged between 7 and 11 years $^{12}$. A representative subsample of 278 children was recruited from the larger cohort to participate in neuroimaging. For the present video game study, we included children with complete cognitive and behavioral evaluation, and excluded statistically-defined 'extreme' video gamers ( $\mathrm{n}=23$ children playing $\geq 18$ hours a week). The final sample comprised 2,442 children (mean age at the baseline, 8.6 years, SD 0.9 and range 7.0 to 11.1 years and including 1,223 boys and 1,219 girls). The neuroimaging subsample comprised 260 children (mean age at baseline, 8.4 years, SD 0.8 and range 7.1 to 10.3 years and including 134 boys and 126 girls) with complete imaging assessment, after the exclusion of statisticallydefined extreme video gamers $(n=3)$.

All parents or tutors signed the informed consent form approved by the Research Ethical Committee (No. 2010/41221/I) of the IMIM-Parc de Salut MAR, Barcelona, Spain and the FP7-ERC-2010-AdG Ethics Review Committee (268479-22022011).

\section{Behavioral measurements}

Video game use. At baseline, parents were asked to estimate how much time (hours) their child was currently playing video games on an average weekday and weekend. A single score was computed from these estimates to indicate average hours of play per week. To document the video games most frequently used, 100 families from the MRI group completed a follow- 
up questionnaire indicating which games were most frequently played in the previous year.

As shown in Table 1, 96\% of children played visuomotor skill-based games (e.g., Super

Mario Bros), full-body active games (exergames; e.g., Wii Sports), or both.

Cognitive testing. The selected cognitive assessment included motor response speed, attention and working memory. Speed of motor responses and attention were assessed using the computerized child version of the "Attentional Network Test" (Child ANT) ${ }^{14}$. Overall reaction time was used to index speed of motor responses to visual stimulation and its standard deviation was used to index motor response consistency as a measurement of sustained attention ${ }^{15}$. Specific visual attention features additionally measured by the ANT task are "alerting", "orienting" and "interference". Commission and omission errors were also registered. Cases with more than $30 \%$ commission or omission errors were excluded from further analyses (total 9 cases in the whole sample and 2 cases in the MRI sample). In the final whole sample, commission errors were $3.1 \%$ and omission errors $1.1 \%$.

A computerized version of the N-Back $\operatorname{task}^{16}$ was used to assess working memory.

"Detectability" (normalized hit rate minus normalized false alarm rate) on 2-back and 3-back loads was used to overall index test performance accuracy. See Forns et al. ${ }^{17}$ for more detail.

Behavioral assessment. Parents completed the Strengths and Difficulties Questionnaire (SDQ) of child behavioral problems ${ }^{18}$. The questionnaire includes 25 questions on psychological attributes, some positive and some negative, which are rated from 0 to 2 points each. Responses are divided in 5 separate scales: "emotional symptoms", “conduct problems", “inattention/hyperactivity", "peer relationship problems" and "prosocial behavior". A "difficulties" score ranging from 0 to 40 was generated by summing the scores for the scales 1 to 4. Parents were also asked to report the average daily sleeping time (hours) of their 
children. Overall school achievement was rated by teachers using a 5-point scale (from the worse $=1$ to the best $=5$ ).

Additional contextual assessments. The neighborhood socioeconomic status vulnerability index (Atlas de Vulnerabilidad Urbana de España, 2012. http://www.fomento.gob.es/... /Atlas Vulnerabilidad Urbana/), at both school and home addresses, was used as a composite measure of socio-demographic factors including level of education, unemployment and occupation at the census tract. Parental education was registered using a 5-point scale (1 illiterate/ 2 less than primary/ 3 primary/ 4 secondary/ 5 university).

\section{MRI acquisition}

MRI was administered a mean $(\mathrm{SD})$ of $1.2( \pm 0.4)$ years after study baseline. A 1.5 Tesla Signa Excite system (General Electric, Milwaukee, WI, USA) equipped with an eight-channel phased-array head coil and single-shot echoplanar imaging (EPI) software was used. The imaging protocol included high-resolution T1-weighted 3D anatomical images, diffusion tensor imaging (DTI) and a 6-min functional MRI sequence acquired in the resting state with eyes closed. Acquisition parameters for the three sequences are fully described in a previous report $^{13}$

\section{Image preprocessing}

\section{Anatomical 3D}

After visual inspection by a trained operator, images from 9 children were discarded as a result of poor image quality and thus the final sample for the anatomical analysis included 251 children. Statistical Parametric Mapping (SPM) voxel-based morphometry (VBM) algorithms with DARTEL registration were used to measure gray and white matter tissue concentration and volume at a voxel level. The preprocessing steps are detailed elsewhere ${ }^{13}$. 
Such a preprocessing approach implied the generation of a study specific template.

Normalized images were transformed to the standard SPM template, re-sliced to $1.5 \mathrm{~mm}$ resolution in Montreal Neurological Institute (MNI) space.

\section{Diffusion tensor imaging (DTI)}

The Functional MRI of the Brain (FMRIB) Software Library 5.0 (FSL), developed by the Analysis Group at the Oxford Centre for FMRIB ${ }^{19}$, was used to process DTI images. Image quality control was rigorous in this study. A total of 86 children were removed from the DTI analysis on the basis of sub-optimal image quality (see Pujol et al. ${ }^{13}$ ). The final DTI sample involved 174 children with a mean $\pm \mathrm{SD}$ of $23.5(94 \%) \pm 1.9$ optimal-quality volumes. Fractional anisotropy (FA) maps were estimated using FDT in FSL after local fitting of the diffusion tensor model at each voxel (“dtifit"). Tract-Based Spatial Statistics ${ }^{20}$ was used to process diffusion data. FA data sets were re-sliced to a $1 \mathrm{~mm} \times 1 \mathrm{~mm} \times 1 \mathrm{~mm}$ anatomical resolution and normalized to standard MNI space via the FMRIB58_FA template and the Non-linear Registration Tool.

\section{Functional MRI}

Preprocessing was carried out using SPM8 and involved motion correction, spatial normalization and smoothing using a Gaussian filter (full-width half-maximum, $8 \mathrm{~mm}$ ). Data were normalized to the standard SPM-EPI template and re-sliced to $2 \mathrm{~mm}$ isotropic resolution in MNI space.

The comprehensive procedures adopted to control for potential head motion effects are fully described in our previous report ${ }^{13}$. In short, the procedures included: (i) Conventional SPM time-series alignment. (ii) Exclusion of 24 children with outlier head motion (mean interframe motion greater than $0.12 \mathrm{~mm}$ ). The finally analyzed sample therefore included 236 children. (iii) The use of both motion-related regressors and estimates of global brain signal 
fluctuations as confounding variables in first-level analyses. (iv) Within-subject, censoringbased MRI signal artifact removal (scrubbing) ${ }^{21,22}$. (v) The use of the mean inter-frame motion across the fMRI run for each participant as a regressor in the second-level analyses ${ }^{22}$.

Based on the results obtained from the anatomical and DTI assessments, our functional connectivity analysis was focused on the left basal ganglia. To systematically explore basal ganglia, we targeted four key subregions: the dorsal and ventral aspects of both caudate nucleus and putamen. Based on a widely applied method for mapping basal ganglia functional connectivity, the regions of interest (or 'seeds') were centered at MNI coordinates (in mm): (i) dorsal caudate nucleus $[\mathrm{x}=-13, \mathrm{y}=15, \mathrm{z}=9]$; (ii) dorsal putamen $[\mathrm{x}=-28, \mathrm{y}=1, \mathrm{z}=3]$; (iii) ventral caudate nucleus, corresponding approximately to the nucleus accumbens $[x=-9, y=9$, $\mathrm{z}=-8]$; and (iv) ventral putamen $[\mathrm{x}=-20, \mathrm{y}=12, \mathrm{z}=-3]$ (see references ${ }^{22-25}$ ).

The procedures adopted to generate functional connectivity maps from the selected regions are detailed in our previous report ${ }^{13}$.

\section{Statistical analysis}

Behavioral data. Student t-test was used to compare sex and age group means as to hours of play per week. ANOVA adjusted by age and sex (ANCOVA) was used to compare gamers and non-gamers as to the behavioral variables. Assumptions under the ANCOVA were met in these analyses including homogeneity of variance, homogeneity of regression slopes of covariates and non-collinearity. Linear regression adjusted by age and sex was used to assess the relationship between video game playing time and behavioral variables within the user group. Bonferroni correction was applied to account for multiple testing within each analysis. Only p-values less than $0.006(0.05 / 8)$ were considered significant. Finally, to easily illustrate the identified associations of gaming with behavior variables, the data were plotted as discrete groups on the basis of playing time in hours (see Figures). 
Imaging analyses. ANOVA with age and sex as covariates (ANCOVA) and crosscorrelations were estimated using SPM. Separate second-level (group) analyses were carried out for whole-brain estimates of regional white matter and gray matter volume, DTI fractional anisotropy and resting-state functional connectivity. The behavioral results obtained in the large sample helped to guide the subsequent imaging analysis. Specifically, three groups of children were defined on the basis of video game use: 45 non-gamers, 113 low use gamers (playing 1-2 hours per week) and 102 high use gamers (playing 3 or more hours per week). The cutoff between low and high use corresponds to the median of time of play per week in all gamers. In each case, ANCOVA allowed us to firstly identify brain areas showing overall differences among these groups. From each analysis, a plot was then generated using brain measurements extracted at representative peak differences in order to characterize the direction of findings with no bias. That is, the three-group model allowed us to fairly capture any change combination among the three groups (non-playing, low use and high use) in a single model sensitive to both the effect of playing vs non-playing and the effect of low versus high use. Finally, post-hoc whole-brain t-tests were used to compare non-gamers with high use gamers. In addition, we mapped voxel-wise the correlation across-subjects between individual brain images and the child's age at the time of MRI acquisition.

Results were considered significant with clusters of $1.032 \mathrm{ml}$ (e.g., 129 voxels with a resolution of $2 \times 2 \times 2 \mathrm{~mm}$ ) at a height threshold of $\mathrm{p}<0.005$, which satisfied the family-wise error (FWE) rate correction of $\mathrm{P}_{\mathrm{FWE}}<0.05$ according to Monte Carlo simulations ${ }^{26}$. 


\section{Results}

\section{Behavioral results}

The overall study sample comprised 428 non-gamers and 2014 gamers (i.e., playing 1 hour or more a week). As a group, gamers played a mean of $4.0 \pm 2.9$ hours a week (Figure 1 for participant distribution according to playing time). Boys overall played video games 1.7 hours a week more than girls (95\% CI, 1.4 to 1.9 hours). There was also a significant effect of age with older children (above sample median age) playing 0.4 hours a week more than younger children ( $95 \%$ CI, 0.2 to 0.7 hours). Although the correlation between age and gaming hours was significant in the whole group $(\mathrm{p}=0.0004)$, collinearity between both variables was very low $(r=0.07$; tolerance $=0.995)$. No significant differences were found between gamers and non-gamers as to parents' education. For example, 59.1\% of mothers of gamers had university-level education versus $61.0 \%$ in non-gamers $\left(\mathrm{Chi}^{2}=0.5 ; \mathrm{p}=0.511\right)$. All further statistical analyses were adjusted for sex and age (i.e., age and sex were included as covariates in both group comparisons and correlations).

Video gamers showed faster motor response to visual stimulation (i.e., shorter reaction time) than non-gamers with a mean group difference of $65 \mathrm{~ms}$ (Table 2). Such a difference was statistically robust $\left(\mathrm{t}=8.1 ; \mathrm{p}=6 \mathrm{e}^{-16}\right)$ and was also significant in the smaller subsample of children who underwent MRI $(\mathrm{t}=4.5 ; \mathrm{p}=0.0001)$. Video game use was also associated with higher consistency of motor responses, measured as reaction time standard deviation (Table 2). However, there were no effects on specific attentional features or working memory. Remarkably, most of the advantage in motor responsiveness was observed in children playing 1 hour per week (mean difference of $52 \mathrm{~ms}$ with respect to non-gamers) with minimal further change observed after 2 hours per week (illustrated in Figure 2). 
By contrast, in the gaming group, the weekly time spent gaming was steadily associated with higher scores in parental ratings of strengths and difficulties (SDQ), particularly in regards to conduct problems (Figure 2), peer conflicts and reduced prosocial abilities (Table 2). In our post-hoc analyses, children gaming in the range of 9-17 hours a week showed significantly more behavioral problems than non-gamers (e.g., conduct problems; $t=3.3$ and $p=0.001$ ). In addition, the amount of time that was spent gaming was negatively associated with sleeping time. However, as a group, gamers and non-gamers did not differ as to strengths and difficulties ratings. Interestingly, gamers showed significantly higher school achievement scores (Table 2).

A further analysis stratified by sex and age showed that the identified associations were notably consistent across the subgroups. In both boys and girls, most of the attained group difference in motor responsiveness was observed playing 1 hour per week, as in the older children subgroup. It is relevant, however, that further improvement was observed up to 3-4 hours of play in the younger children. In each stratified group, differences between gamers and non-gamers were highly significant (boys $\mathrm{p}=2 * \mathrm{e}^{-10}$; girls $\mathrm{p}=5^{*} \mathrm{e}^{-8}$; younger $\mathrm{p}=3 * \mathrm{e}^{-10}$; and older $\mathrm{p}=9 * \mathrm{e}^{-9}$ ). However, such differences between gamers and non-gamers were significantly stronger in girls compared to boys (interaction $\mathrm{t}=2.6 ; \mathrm{p}=0.008$ ). No significant interaction was identified for age. Similarly, gamers showed a significant linear correlation between playing hours and conduct problems in each subgroup analysis (boys $p=0.001$; girls $\mathrm{p}=1 * \mathrm{e}^{-5}$; younger $\mathrm{p}=8^{*} \mathrm{e}^{-6}$; and older $\left.\mathrm{p}=0.002\right)$. No significant interaction was found in the correlation analysis. Also, we found no significant differences between gamers and nongamers in any subgroup (boys, girls, younger and older children) as to conduct problems. All associations remained highly significant when analyses were further adjusted for other potential confounding variables, including 'parental education' and 'neighborhood vulnerability index' as the most representative indicators of children's close environment. 


\section{Imaging results}

Age and sex were included as covariates in all imaging analyses.

T1-weighted 3D anatomical images. When examining differences among non-gamers and low and high use gamers in white matter volume measurements (ANCOVA), significant results were observed in a region adjacent to the ventral and lateral aspect of the striatum and extending to the temporal lobe (Figure 3 and Supplementary eTable 1). A plot of white matter volume measurements from the left ventral striatal region revealed a similar increase of white matter volume in both low- and high-user groups compared with non-users. Figure 3 illustrates this effect in post-hoc comparison between non-users and high users. In addition, the primary ANCOVA identified significant effects in the brainstem (Supplementary eTable 1), which were not observed in the post-hoc analysis. No significant results were obtained for gray matter volumes.

\section{Diffusion tensor imaging (DTI) fractional anisotropy (FA). ANCOVA consistently} showed significant differences among groups in a region adjacent to the lateral aspect of the striatum (Figure 3, Supplementary eTable 1). The plot of FA measurements from this left striatal region revealed a relevant FA increase, but only in the high-use group (Figure 3). Direct comparison of non-gamers with high-use gamers confirmed the increase of FA in this region and detected additional significant changes in the ventral striatal region bilaterally, as well as the right thalamus and left occipital white matter.

Basal ganglia functional connectivity. Video gaming was associated with higher functional connectivity in the putamen and caudate nucleus maps (Figure 4 and Supplementary eTable 1). Specifically in the putamen maps, a significant connectivity increase was identified with the left motor cortex and prefrontal cortex in both low and high user groups. In the ventral caudate map, the most relevant finding involved the dorsal anterior cingulate cortex (ACC), 
which demonstrated a strong effect in the high-use group and an intermediate effect in the low-use group compared with non-gamers (Figure 4). The post-hoc analysis confirmed a robust connectivity increase with the ACC and additionally with the left and right anterior insula region (Figure 4 and Supplementary eTable 1).

Correlation analysis with age (illustrated in Figure 5). White matter volume increased with age in the visual system and sensory-motor projection tracts at the level of basal ganglia and thalamus, with no areas showing negative correlations with age. FA increased significantly with age mostly in the internal capsule and basal ganglia. Therefore, the identified associations of video gaming with white matter measurements were generally consistent with the effects of age in terms of their positive direction and (partial) anatomical overlap.

Similarly, age was associated with higher functional connectivity between the putamen and motor cortex in the area associated with video game use, albeit significant only for right hemispheric effects. For the ventral caudate, age was instead negatively associated with its functional connectivity with a medial frontal area adjacent to the ACC region that was positively associated with gaming use (and additionally with the upper brainstem and amygdala).

\section{Discussion}

In the cognitive domain, video game use was associated with faster motor response to visual stimulation. Importantly, no further change in motor speed was identified in children playing more than 2 hours a week, suggesting an early ceiling effect for this measurement. Video gamers, overall, did not demonstrate more problematic behavior that non-gamers, although, within the gaming group, we observed a positive association between time weekly spent gaming and conduct problems, peer conflicts and reduced prosocial abilities, as well as a 
negative association with sleeping time. At a neural level, structural and functional brain changes associated with gaming use were most evident with respect to basal ganglia circuitry.

Our cognitive assessment selectively measured processing speed, attention and working memory as primary cognitive domains of interest. In the literature, there is some consensus that gaming may improve speed of information processing and motor response $e^{3,27,28}$, however, the reported effects on executive function and working memory, as well as general intelligence, have been less consistent ${ }^{3}$. For example, working memory has been shown to improve in some studies, whereas others reported only partial associations or negative results $^{29-31}$. In our study, larger differences in reaction time than in working memory supports the contention that several abilities are more trainable than others with commercial video $\operatorname{games}^{3,7,8}$.

(cose

We observed an association between gaming use and changes in basal ganglia circuits in the form of structural (white matter) and functional connectivity increases. These findings are intuitive in many respects, as it is well known that basal ganglia circuits are critical for procedural learning based on the acquisition of new skills through practice ${ }^{7-9}$. Our results are also generally consistent with other imaging studies reporting significant effects of gaming use on the fronto-basal ganglia system structure and function ${ }^{32-37}$. Specifically, Erickson et al. ${ }^{37}$ showed that the acquisition of skills on demanding video games may be predicted by variations in the volume of the striatum. Children traditionally acquire procedural skills through action, for instance in relation to sports and outdoor games. Neuroimaging research now suggests that training with desktop virtual environments is also capable of modulating brain systems that support procedural learning. However, the type of video game is likely to be particularly relevant with regards to the modulation of specific neural systems. For example, the use of logic and "platform jumping" games has been associated with anatomical changes implicating the hippocampal system in adults ${ }^{38,39}$. 
Reviews of the literature generally conclude that excessive video game use has negative effects on some aspects of behavior in children ${ }^{1,3,5,40}$. Most of the identified problems relate to the extent to which gaming can become a behavioral addiction, or with regards to the violent contents of some of the games themselves. We did not include children with probable gaming addiction and the amount of time spent on playing in our population (mean 4 hours a week, range 0-17 hours) was far less than the gaming level that has been associated with severe psychosocial health disturbances. Despite this, gaming use in our study was positively related to the presence of conduct problems, peer conflicts and reduced prosocial abilities. This finding is in agreement with the results of a national survey of 10 to 15 years old children in the UK, in which gaming use of more than 3 hours per day was associated with similar behavioral problems ${ }^{41}$. Thus, frequent video game use does appear to be associated with behavioral problems in children even in absence of recognized gaming disorder, although the direction of the relationship is less clear ${ }^{6}$. That is, children with peer conflicts and reduced prosocial abilities may show a tendency to isolate and spend more time playing video games. On the other hand, highly frequent use likely consumes a proportion of the child's leisure time and invades other activities that may ultimately affect the normal development of prosocial abilities.

Our study was limited in that we applied a selective cognitive and behavioral assessment that did not comprehensively cover all domains. Also, we estimated the time spent gaming from parental reports but with no formal assessment of the fidelity of these reports in terms of their reliability and accuracy. It is also relevant to mention that the estimation of video game use was based on a single cross-sectional measurement. Finally, the type of video games played varied and we were unable to perform specific analyses stratified by game content or type.

In conclusion, we have investigated relationships between weekly video game use, selected cognitive abilities, conduct-related problems and brain correlates in a large group of children. 
Relatively small amounts of video gaming was associated with better performance in certain visuo-motor skills and, by contrast, a frequent use was associated with the presence of conduct problems. Whereas motor response differences between gamers and no-gamers were already established with 2 hours a week of video gaming, conduct problems appear to be morecom more common in school children weekly playing 9 hours or more. It is important to emphasize that this is a correlational study and, as such, does not permit direct inferences regarding causal relationships. Further studies will be needed to explore the extent to which better visuo-motor skills and conduct problems are a cause or consequence of gaming.
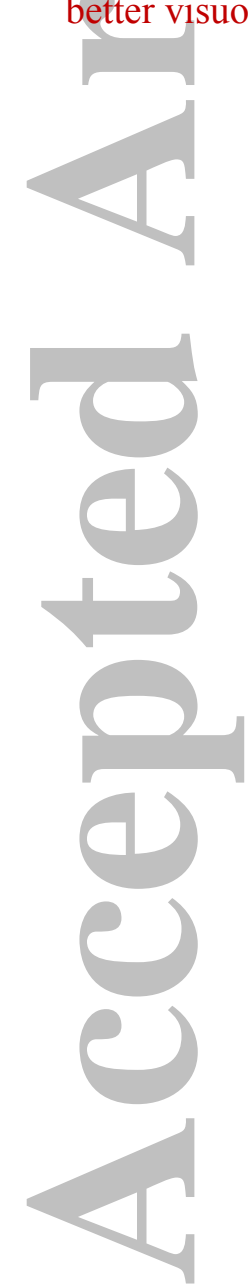


\section{Acknowledgments}

This work was supported by the European Research Council under the ERC [grant number 268479] - the BREATHE project. The Agency of University and Research Funding Management of the Catalonia Government participated in the context of Research Group SGR2014-1673. We acknowledge Cecilia Persavento, Judit González, Laura Bouso, Mónica López and Pere Figueras for their contribution to the field work. We also acknowledge all the families and schools participating in the study.

Author Contributions: JP, JS, LBH and JD contributed to the study concept and design; JP, MAP, JF, SGO, RF, DM and GMV contributed to data acquisition and analysis; and JP and BJH contributed to drafting the manuscript and figures.

\section{Conflicts of interest}

The authors declare no conflict of interest.

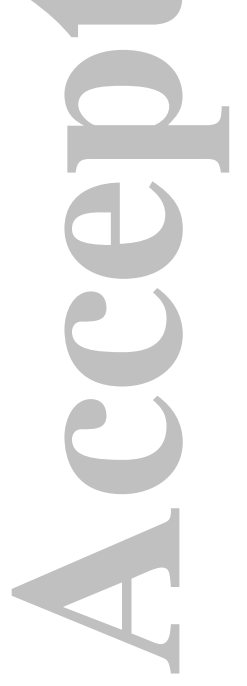




\section{REFERENCES}

1. Bavelier D, Green CS, Han DH, et al. Brains on video games. Nat Rev Neurosci. $2011 ; 12(12): 763-768$.

2. Ferguson CJ. Do Angry Birds Make for Angry Children? A Meta-Analysis of Video Game Influences on Children's and Adolescents' Aggression, Mental Health, Prosocial Behavior, and Academic Performance. Perspect Psychol Sci. 2015;10(5):646-666.

\section{Powers KL, Brooks PJ, Aldrich NJ, et al. Effects of video-game play on information} processing: a meta-analytic investigation. Psychon Bull Rev. 2013 Dec;(6):1055-1079.

4. Bavelier D, Green CS, Pouget A, Schrater P. Brain plasticity through the life span: learning to learn and action video games. Annu Rev Neurosci. 2012;35:391-416.

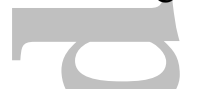

5. Anderson CA, Shibuya A, Ihori N, et al. Violent video game effects on aggression, empathy, and prosocial behavior in eastern and western countries: a meta-analytic review.

Psychol Bull. 2010;136(2):151-173.

6. Gentile DA, Choo H, Liau A, et al. Pathological video game use among youths: a twoyear longitudinal study. Pediatrics. 2011;127(2):e319-329.

7. Melby-Lervåg M, Hulme C. Is working memory training effective? A meta-analytic review. Dev Psychol. 2013;49(2):270-291.

8. Shipstead Z1, Redick TS, Engle RW. Is working memory training effective? Psychol Bull. 2012;138(4):628-654.

9. Doyon J, Penhune V, Ungerleider LG. Distinct contribution of the cortico-striatal and cortico-cerebellar systems to motor skill learning. Neuropsychologia. 2003;41(3):252-262. 
10. Graybiel AM. Habits, rituals, and the evaluative brain. Annu Rev Neurosci. 2008;31:359-387.

11. Yin HH, Knowlton BJ. The role of the basal ganglia in habit formation. Nat Rev Neurosci. 2006;7(6):464-476.

12. Sunyer J. Esnaola M, Alvarez-Pedrerol M, et al. Traffic-related air pollution in schools impairs cognitive development in primary school children. PLoS Med. 2015;2: e1001792.

13. Pujol J, Martínez-Vilavella G, Macià D et al. Traffic pollution exposure is associated with altered brain connectivity in school children. Neuroimage. 2016;129:175-184.

14. Rueda MR, Fan J, McCandliss BD, et al. Development of attentional networks in childhood. Neuropsychologia. 2004;42(8):1029-1040.

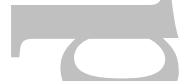

15. Langner R, Eickhoff SB. Sustaining attention to simple tasks: a meta-analytic review of the neural mechanisms of vigilant attention. Psychol. Bull. 2013;139(4):870-900.

16. Anderson P. Assessment and development of executive function (EF) during childhood. Child Neuropsychology. 2002;8(2):71-82.

17. Forns J, Esnaola M, López-Vicente M, et al. The n-back test and the attentional network task as measures of child neuropsychological development in epidemiological studies. Neuropsychology. 2014;28(4):519-529.

18. Goodman R. Psychometric properties of the strengths and difficulties questionnaire. $J$ Am Acad Child Adol Psychiatry. 2001;40(11):1337-1345.

19. Smith SM, Jenkinson M, Woolrich MW, et al. Advances in functional and structural MR image analysis and implementation as FSL. Neuroimage. 2004;23 Suppl 1:S208-219. 
20. Smith SM, Jenkinson M, Johansen-Berg H, et al. Tract-based spatial statistics: voxelwise analysis of multi-subject diffusion data. Neuroimage. 2006;31(4):1487-1505.

21. Power JD, Mitra A, Laumann TO, et al. Methods to detect, characterize, and remove motion artifact in resting state fMRI. Neuroimage. 2014;84:320-341.

22. Pujol J, Macià D, Blanco-Hinojo L, et al. Does motion-related brain functional connectivity reflect both artifacts and genuine neural activity? Neuroimage. 2014;101: 87-95.

23. Di Martino A, Fair DA, Kelly C, et al. Unraveling the miswired connectome: a developmental perspective. Neuron. 2014;83(6):1335-1353.

24. Harrison BJ, Soriano-Mas C, Pujol J, et al. Altered corticostriatal functional connectivity in obsessive-compulsive disorder. Arch Gen Psychiatry. 2009;66(11):1189-1200.

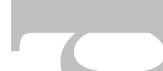

25. Harrison BJ, Pujol J, Cardoner N, et al. Brain corticostriatal systems and the major clinical symptom dimensions of obsessive-compulsive disorder. Biol Psychiatry.

$2013 ; 73(4): 321-328$.

26. Pujol J, Macià D, Garcia-Fontanals A, et al. The contribution of sensory system functional connectivity reduction to clinical pain in fibromyalgia. Pain. 2014;155(8): 14921503.

27. Latham AJ, Patston LL, Tippett LJ. The virtual brain: 30 years of video-game play and cognitive abilities. Front Psychol. 2013 Sep 13;4:629

28. Dye MW, Green CS, Bavelier D. Increasing speed of processing with action video games. Curr Dir Psychol Sci. 2009;18(6):321-326.

29. Oei AC, Patterson MD. Enhancing cognition with video games: a multiple game training study. PLoS One. 2013;8(3):e58546. 
30. Blacker KJ, Curby KM, Klobusicky E, Chein JM. Effects of action video game training on visual working memory. J Exp Psychol Hum Percept Perform. 2014;40(5):19922004.

31. Boot WR, Kramer AF, Simons DJ, et al. The effects of video game playing on attention, memory, and executive control. Acta Psychol (Amst). 2008;129(3):387-398.

32. Kim YH, Kang DW, Kim D, et al. Real-time strategy video game experience and visual perceptual learning. J Neurosci. 2015;35(29):10485-10492.

33. Lorenz RC, Gleich T, Gallinat J, Kühn S. Video game training and the reward system. Front Hum Neurosci. 2015;9:40.

34. Kühn S, Romanowski A, Schilling C, et al. The neural basis of video gaming. Transl Psychiatry. 2011;1:e53.

35. Kühn S, Lorenz R, Banaschewski T, et al. Positive association of video game playing with left frontal cortical thickness in adolescents. PLoS One. 2014;9(3):e91506.

36. Hyun GJ, Shin YW, Kim BN, et al. Increased cortical thickness in professional on-line gamers. Psychiatry Investig. 2013;10(4):388-392.

37. Erickson KI, Boot WR, Basak C, et al. Striatal volume predicts level of video game skill acquisition. Cereb Cortex. 2010;20(11):2522-2530.

38. Kühn S, Gleich T, Lorenz RC, et al. Playing Super Mario induces structural brain plasticity: gray matter changes resulting from training with a commercial video game. Mol Psychiatry. 2014;19(2):265-271.

39. Kühn S, Gallinat J. Amount of lifetime video gaming is positively associated with entorhinal, hippocampal and occipital volume. Mol Psychiatry. 2014;19(7):842-847. 
40. Straker L, Abbott R, Collins R, Campbell A. Evidence-based guidelines for wise use of electronic games by children. Ergonomics. 2014;57(4):471-489.

41. Przybylski AK. Electronic gaming and psychosocial adjustment. Pediatrics. 2014;134(3):e716-22.
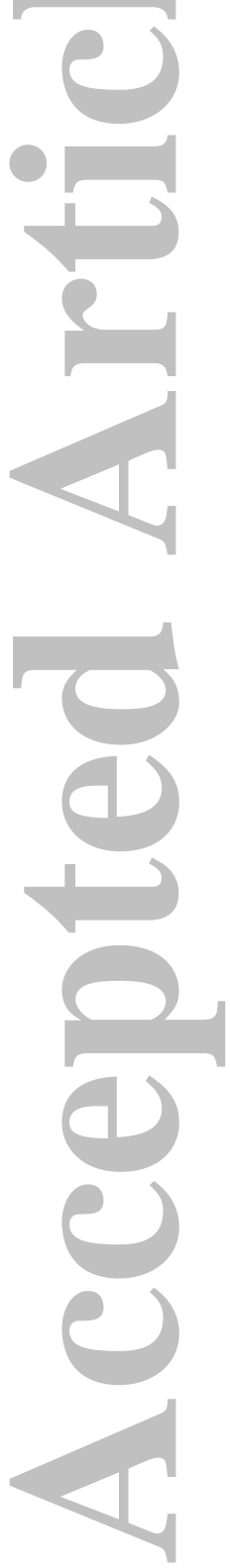


\section{Figure legends}

Figure 1. Participant distribution according to playing time.

Figure 2. Mean reaction time (top) and conduct scores (bottom) plotted for discrete playing time groups. Both plots are adjusted by age and sex and display $95 \%$ confidence interval (CI) estimates.

Figure 3. Gaming group differences in white matter volume measurements (top left) and diffusion tensor imaging (DTI) fractional anisotropy (FA) (top right). ANOVA adjusted by age and sex (ANCOVA) indicated significant changes adjacent to the striatum in both analyses. Mean and 95\% CIs are plotted for each modality, reflecting region peak differences among gaming groups. The bottom left and right images illustrate corresponding differences identified between non-gamers versus high use gamers. The left hemisphere corresponds to the left side of coronal views.

Figure 4. Gaming group differences in basal ganglia functional connectivity. ANCOVA indicated significant changes in functional connectivity between the dorsal putamen (DP) and motor cortex at the level of the frontal operculum. In the ventral caudate (VC) analysis, the most relevant finding involved the anterior cingulate cortex. Mean and 95\% CIs are plotted, reflecting region peak differences among gaming groups. The bottom left and right images illustrate corresponding differences identified between non-gamers versus high use gamers. The left hemisphere corresponds to the left side of axial views.

Figure 5. Correlations between age and brain measurements. White matter volume (a) increased significantly with age in projection tracts (and the visual system). Fractional anisotropy (b) increased with age mostly in the internal capsule and basal ganglia. Age was 
also associated with stronger functional connectivity between the putamen and motor cortex (c) and weaker functional connectivity between the caudate nucleus and the medial frontal cortex (d) and additionally with the upper brainstem (and amygdala).
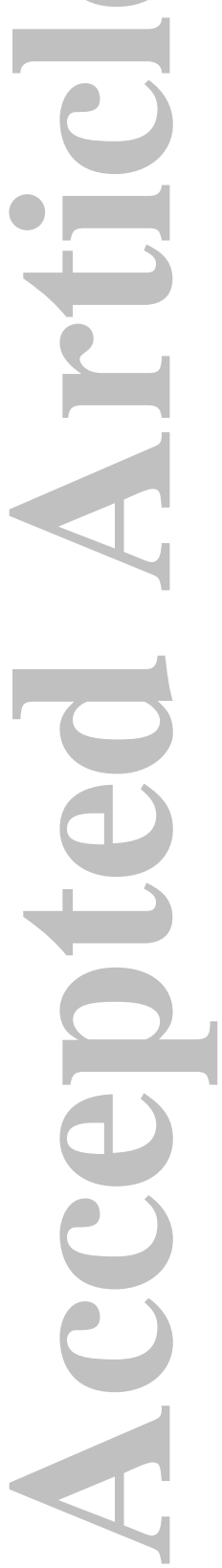
Table 1. Frequency of video games used (in \%).

\section{Visuomotor skill-based games (alone or combined): $\mathbf{7 8 \%}$}

Platforms: Mario Bros/SuperMario Bros/Luigi/Super Mario Galaxy (46\%), Donkey Kong (12\%) Sports simulation: Fifa (33\%), Pro-evolution soccer (8\%), Inazuma eleven (8\%), NBA (4\%) Driving simulation: Mario Karts (7\%), Formula 1 (3\%), Gran Turismo (2\%)

Shooting: Call of Duty (2\%)

Fighting: Dragon Ball $(2 \%)$

Adventure with Action: Lego city (5\%), Grand Theft Auto (3\%)

Full-body active games- exergames (alone or combined): $\mathbf{5 8 \%}$

Wii sports (48\%), Just Dance (20\%), Mario Party (17\%), Wii Party (4\%)

\section{Adventure/Strategy games (alone or combined): $49 \%$}

Animal Crossing (12\%), Pokemon (11\%), Minecraft (6\%), Dogz/Horsez (4\%), others (12\%)

Visuomotor skill-based games and/or Full-body active games; 96\% Adventure/Strategy games alone; $4 \%$

Based on reports from 100 families indicating up to three games played the most in the year before MRI. 
Pujol 26

Table 2. Behavioral results

\begin{tabular}{|c|c|c|c|c|c|c|c|c|}
\hline & \multicolumn{5}{|c|}{ Group Comparison } & \multicolumn{3}{|c|}{$\begin{array}{c}\text { Correlation with } \\
\text { Weekly Play Time }\end{array}$} \\
\hline & $\begin{array}{c}\text { Non-users } \\
\mathrm{n}=428\end{array}$ & $\begin{array}{c}\text { Users } \\
\mathrm{n}=2014\end{array}$ & & & & & $\begin{array}{l}\text { sers } \\
2014)\end{array}$ & \\
\hline Cognitive Tests & $\begin{array}{l}\text { Adj. Mean } \\
95 \% \text { CI }\end{array}$ & $\begin{array}{c}\text { Adj Mean } \\
95 \% \text { CI }\end{array}$ & $\begin{array}{l}\text { Diff. } \\
95 \% \mathrm{CI}\end{array}$ & $\mathrm{t}$ & $\mathrm{p}$ & $\beta$ & $\mathrm{t}$ & $\mathrm{p}$ \\
\hline Reaction Time (ms) & $\begin{array}{c}861 \\
847 / 875\end{array}$ & $\begin{array}{c}796 \\
790 / 802\end{array}$ & $\begin{array}{c}65 \\
49 / 81\end{array}$ & 8.1 & $6 e^{-16}$ & -0.559 & -0.5 & 0.619 \\
\hline Reaction Time SD (ms) & $\begin{array}{c}286 \\
278 / 294\end{array}$ & $\begin{array}{c}267 \\
264 / 271\end{array}$ & $\begin{array}{c}19 \\
10 / 28\end{array}$ & 4.0 & 0.00005 & -0.201 & -0.3 & 0.761 \\
\hline Alerting (ms) & $\begin{array}{c}43 \\
35 / 50\end{array}$ & $\begin{array}{c}49 \\
46 / 53\end{array}$ & $\begin{array}{c}-6 \\
-15 / 2\end{array}$ & -1.5 & 0.128 & 1.161 & 2.0 & 0.045 \\
\hline Orienting (ms) & $\begin{array}{c}32 \\
25-40\end{array}$ & $\begin{array}{c}35 \\
31 / 38\end{array}$ & $\begin{array}{c}-3 \\
-11 / 6\end{array}$ & -0.6 & 0.575 & -1.102 & -2.0 & 0.057 \\
\hline Interference (ms) & $\begin{array}{c}66 \\
60 / 72\end{array}$ & $\begin{array}{c}63 \\
60 / 66\end{array}$ & $\begin{array}{c}3 \\
-3 / 10\end{array}$ & 1.0 & 0.340 & 1.082 & 2.3 & 0.021 \\
\hline 2B Working Mem. (d') & $\begin{array}{c}2.2 \\
2.1-2.3\end{array}$ & $\begin{array}{c}2.3 \\
2.2 / 2.3\end{array}$ & $\begin{array}{c}-0.08 \\
-0.2 / 0.05\end{array}$ & -1.2 & 0.229 & -0.015 & -1.6 & 0.118 \\
\hline 3B Working Mem.(d') & $\begin{array}{c}1.1 \\
1.0 / 1.2^{\mathrm{a}}\end{array}$ & $\begin{array}{c}1.2 \\
1.2 / 1.3^{\mathrm{b}}\end{array}$ & $\begin{array}{c}-0.07 \\
-0.2 / 0.04\end{array}$ & -1.3 & 0.210 & -0.012 & -1.5 & 0.124 \\
\hline School Achievement & $\begin{array}{c}3.41 \\
3.31 / 3.51\end{array}$ & $\begin{array}{c}3.60 \\
3.55 / 3.64\end{array}$ & $\begin{array}{c}-0.19 \\
-0.26 /-0.03\end{array}$ & -3.2 & 0.001 & -0.002 & -0.3 & 0.780 \\
\hline Sleeping Time (hours) & $\begin{array}{c}9.63 \\
9.56 / 9.7\end{array}$ & $\begin{array}{c}9.56 \\
9.53 / 9.59\end{array}$ & $\begin{array}{c}0.07 \\
-0.007 / 0.1\end{array}$ & 1.8 & 0.076 & -0.029 & -5.3 & $1 e^{-7}$ \\
\hline Total SDQ score & $\begin{array}{c}8.6 \\
8.1 / 9.1\end{array}$ & $\begin{array}{c}8.3 \\
8.1 / 8.6\end{array}$ & $\begin{array}{c}0.27 \\
-0.30 / 0.84\end{array}$ & 0.9 & 0.353 & 0.188 & 4.6 & $5 e^{-6}$ \\
\hline Conduct Problems & $\begin{array}{l}1.7 \\
1.5 / 1.8\end{array}$ & $\begin{array}{c}1.6 \\
1.6 / 1.7\end{array}$ & $\begin{array}{c}0.04 \\
-0.13 / 0.21\end{array}$ & 0.4 & 0.661 & 0.065 & 5.4 & $8 e^{-8}$ \\
\hline Problems with Peer & $\begin{array}{c}1.2 \\
1.0 / 1.3\end{array}$ & $\begin{array}{c}1.1 \\
1.1 / 1.2\end{array}$ & $\begin{array}{c}0.07 \\
-0.09 / 0.23\end{array}$ & 0.8 & 0.420 & 0.061 & 5.2 & $2 e^{-7}$ \\
\hline Emotional Symptoms & $\begin{array}{c}1.9 \\
1.7 / 2.1\end{array}$ & $\begin{array}{c}1.9 \\
1.9 / 2.0\end{array}$ & $\begin{array}{c}-0.03 \\
-0.22 / 0.17\end{array}$ & -0.3 & 0.787 & 0.030 & 2.1 & 0.033 \\
\hline Inattention/Hyperac. & $\begin{array}{c}3.8 \\
3.6 / 4.1\end{array}$ & $\begin{array}{c}3.7 \\
3.6 / 3.8\end{array}$ & $\begin{array}{c}0.19 \\
-0.08 / 0.46\end{array}$ & 1.4 & 0.169 & 0.033 & 1.7 & 0.094 \\
\hline Prosocial Behavior & $\begin{array}{c}8.5 \\
8.3 / 8.7\end{array}$ & $\begin{array}{c}8.5 \\
8.4 / 8.6\end{array}$ & $\begin{array}{c}<-0.006 \\
-0.18 / 0.16\end{array}$ & -0.1 & 0.941 & -0.039 & -3.2 & 0.002 \\
\hline
\end{tabular}


All the analyses were adjusted by sex and age. Adj., adjusted. SD, standard deviation. Bold indicates significant after Bonferroni correction. 2B and 3B indicates 2-back and 3-back, respectively. a, $n=408$. b, n=1967. "d"”, detectability. SDQ, Strengths and Difficulties Questionnaire.
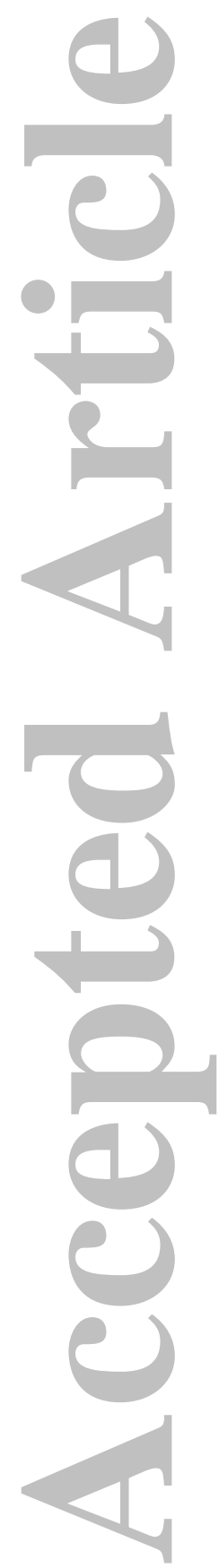


\section{Supplementary Material}

Supplementary eTable 1. MRI results

\section{Cluster size, $m l \quad x y z$}

\section{White Matter Volume ANCOVA}

L Ventral Striatum Region-Anterior temporal

1.2

$-293-15$

$F=7.2$

Posterior Mesencephalon

1.4

$-3-39-18$

$\mathrm{F}=6.3$

Anterior Pons

1.1

$3-12-30 \quad F=6.9$

Post-Hoc High Users > Non-Users

L Ventral Striatum Region-Anterior temporal

6.1

$-305-14 \quad \mathrm{t}=3.7$

DTI Fractional Anisotropy ANCOVA

L Lateral Striatum Region

Post-Hoc High Users $>$ Non-Users

L Lateral Striatum Region

L Ventral Striatum Region

R Ventral Striatum Region

R Thalamus

C

L Occipital Lobe

\section{Functional Connectivity}

Dorsal Putamen Map ANCOVA

L Motor Cortex

\section{Post-Hoc High Users $>$ Non-Users}

L Motor Cortex

Ventral Putamen Map ANCOVA

L Lateral Prefrontal Cortex

Medial Frontal Cortex
1.4

$-28216$

$\mathrm{F}=9.6$

1.5

1.5

1.4

1.7

1.9

$-28414 \quad \mathrm{t}=3.8$

$-1317-6 \quad t=4.0$

$1119-7 \quad \mathrm{t}=3.7$

$16-2211 \quad \mathrm{t}=3.9$

$-24-684 \quad t=3.7$

1.2

$$
-50-418 \quad F=7-7
$$

6.7

$-50-418 \quad \mathrm{t}=3.9$

1.9

$-423218$

$\mathrm{F}=9.8$

1.3

$-21650 \quad \mathrm{~F}=7.1$ 
Pujol 29

\section{Post-Hoc High Users > Non-Users}

\begin{tabular}{|c|c|c|c|}
\hline L Lateral Prefrontal Cortex & 4.0 & -423418 & $\mathrm{t}=4.1$ \\
\hline L Motor Cortex & 1.2 & $-58-414$ & $\mathrm{t}=3.3$ \\
\hline Ventral Caudat & & & \\
\hline Anterior Cingulate Cortex & 4.0 & 41234 & $\mathrm{~F}=10.6$ \\
\hline L Parietal Operculum & 2.2 & $48-3018$ & $F=13.9$ \\
\hline Post-Hoc High U & & & \\
\hline Anterior Cingulate Cortex & 9.9 & 61034 & $\mathrm{t}=4.5$ \\
\hline R Anterior Insula Region & 6.1 & 32160 & $\mathrm{t}=4.0$ \\
\hline L. Anterior Insula Region & 2.0 & -40126 & $\mathrm{t}=3.7$ \\
\hline
\end{tabular}

$\mathrm{x}$ y z, coordinates given in Montreal Neurological Institute (MNI) space. Statistics at corrected threshold $\mathrm{P}_{\mathrm{FWE}}$ $<0.05$ estimated using Monte Carlo simulations. 

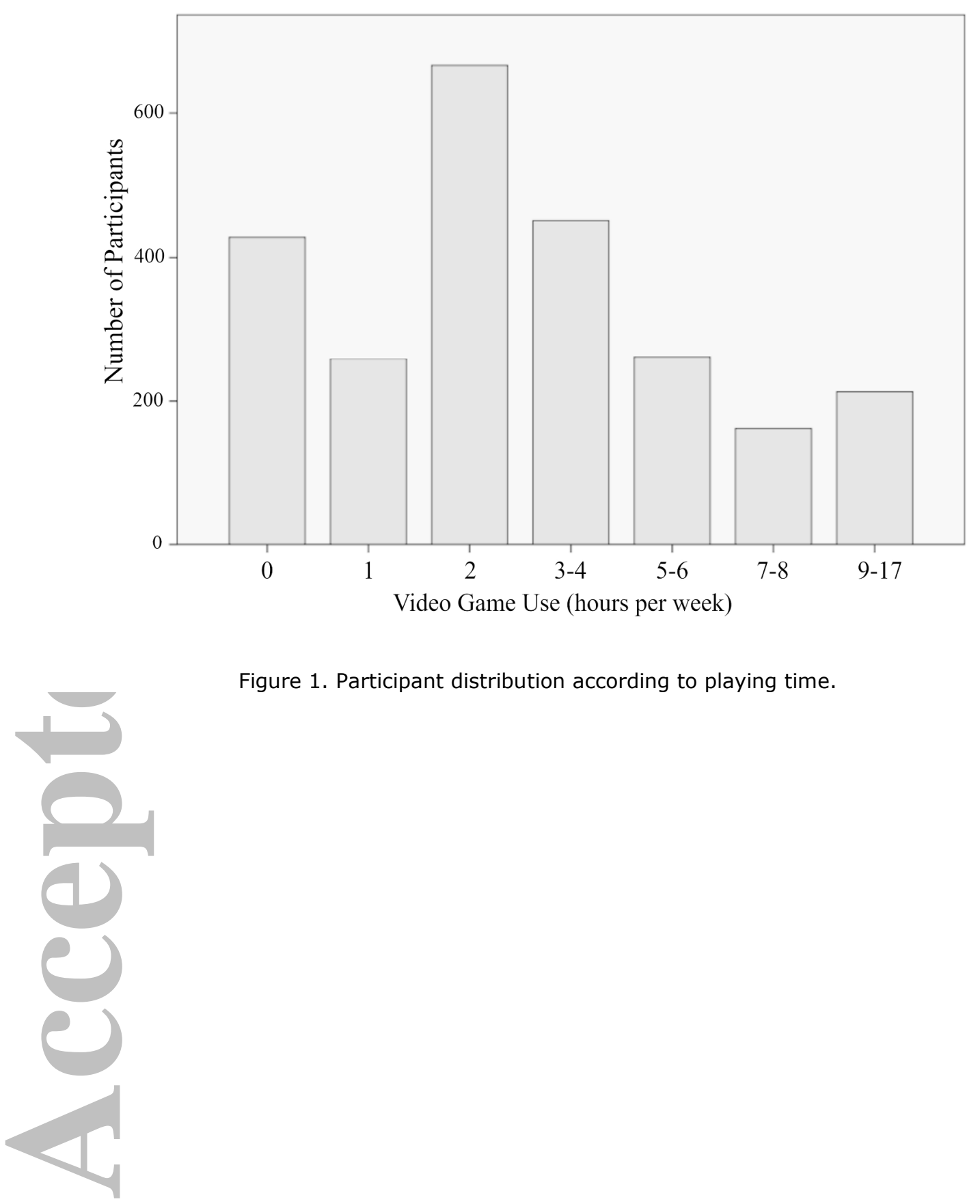

Figure 1. Participant distribution according to playing time. 

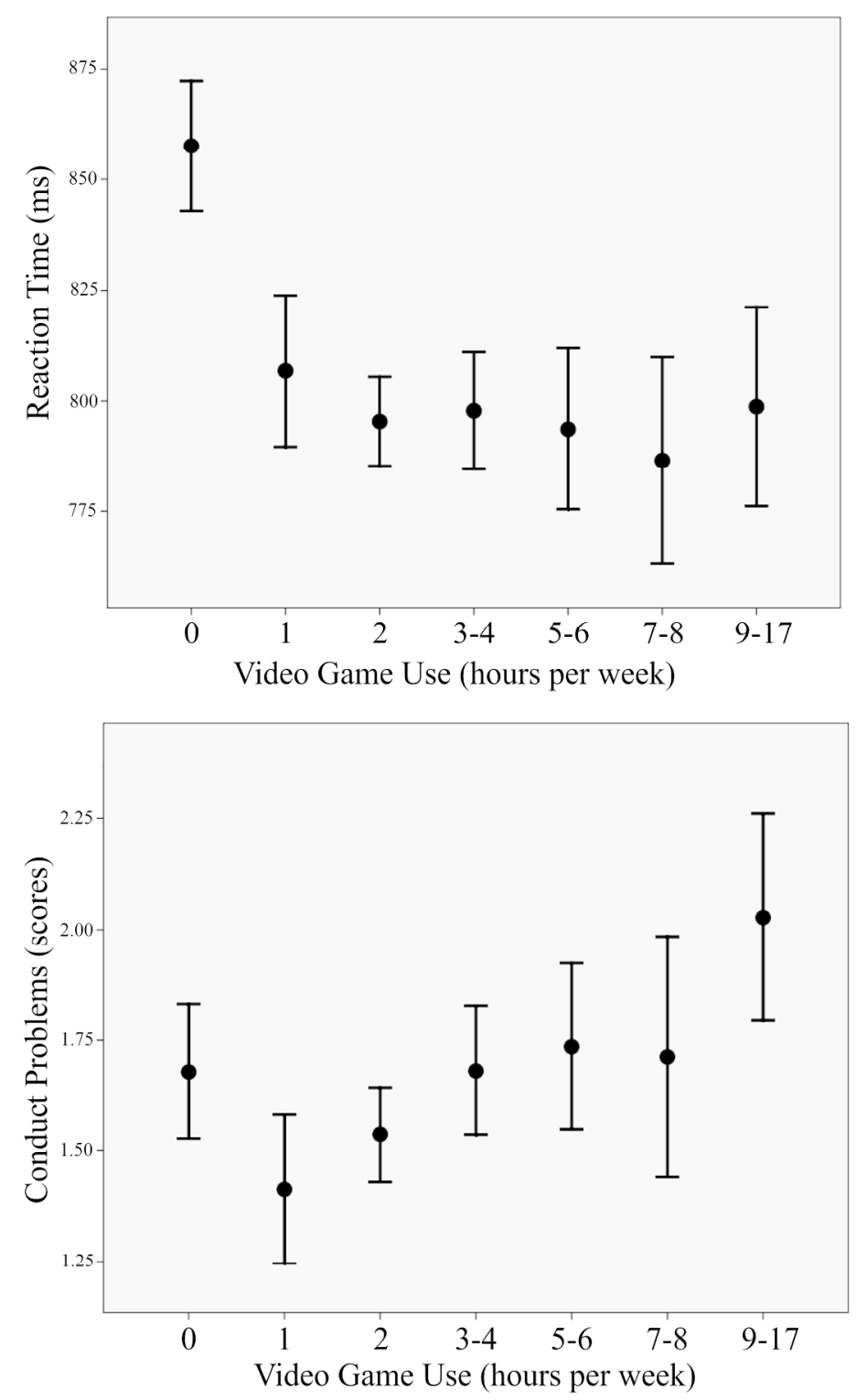

Figure 2. Mean reaction time (top) and conduct scores (bottom) plotted for discrete playing time groups. Both plots are adjusted by age and sex and display $95 \%$ confidence interval (CI) estimates. 

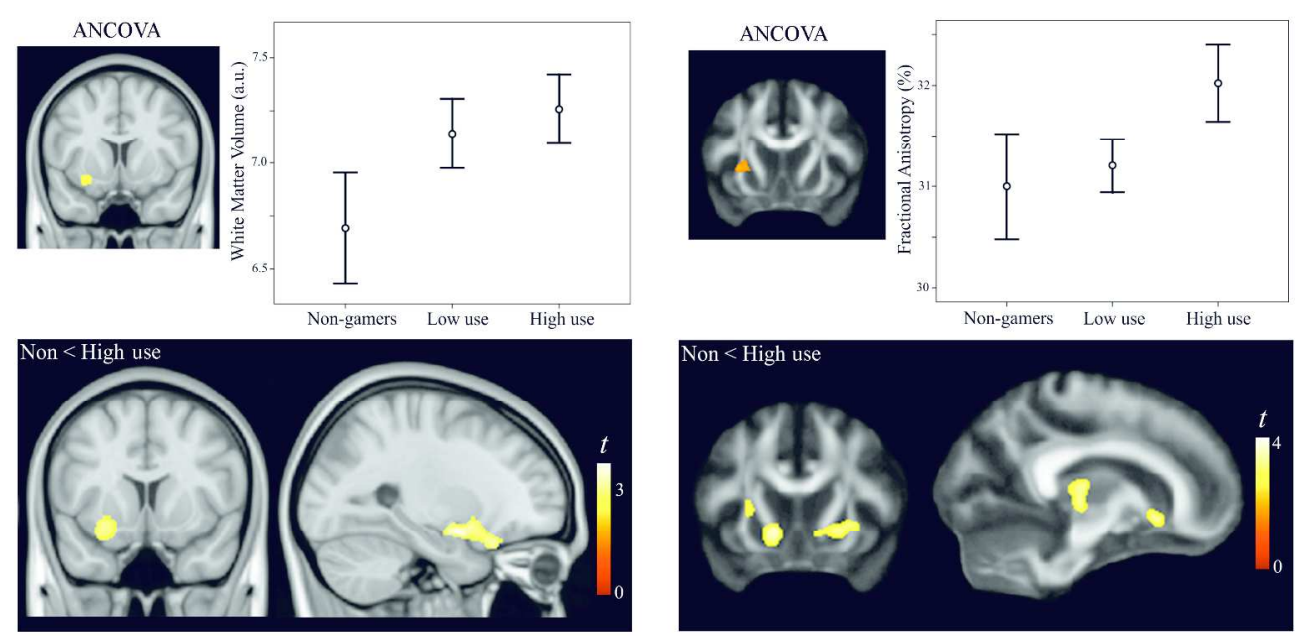

Figure 3. Gaming group differences in white matter volume measurements (top left) and diffusion tensor imaging (DTI) fractional anisotropy (FA) (top right). ANOVA adjusted by age and sex (ANCOVA) indicated significant changes adjacent to the striatum in both analyses. Mean and $95 \%$ CIs are plotted for each modality, reflecting region peak differences among gaming groups. The bottom left and right images illustrate corresponding differences identified between non-gamers versus high use gamers. The left hemisphere corresponds to the left side of coronal views.

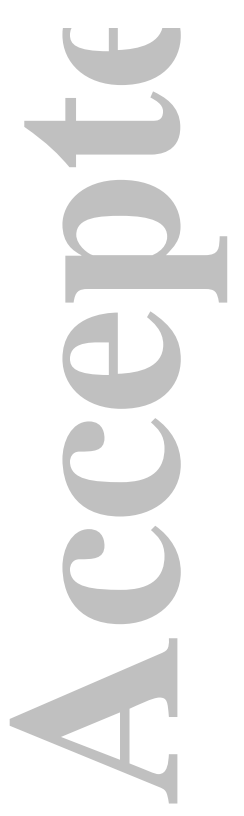

$254 \times 127 \mathrm{~mm}(300 \times 300$ DPI $)$ 

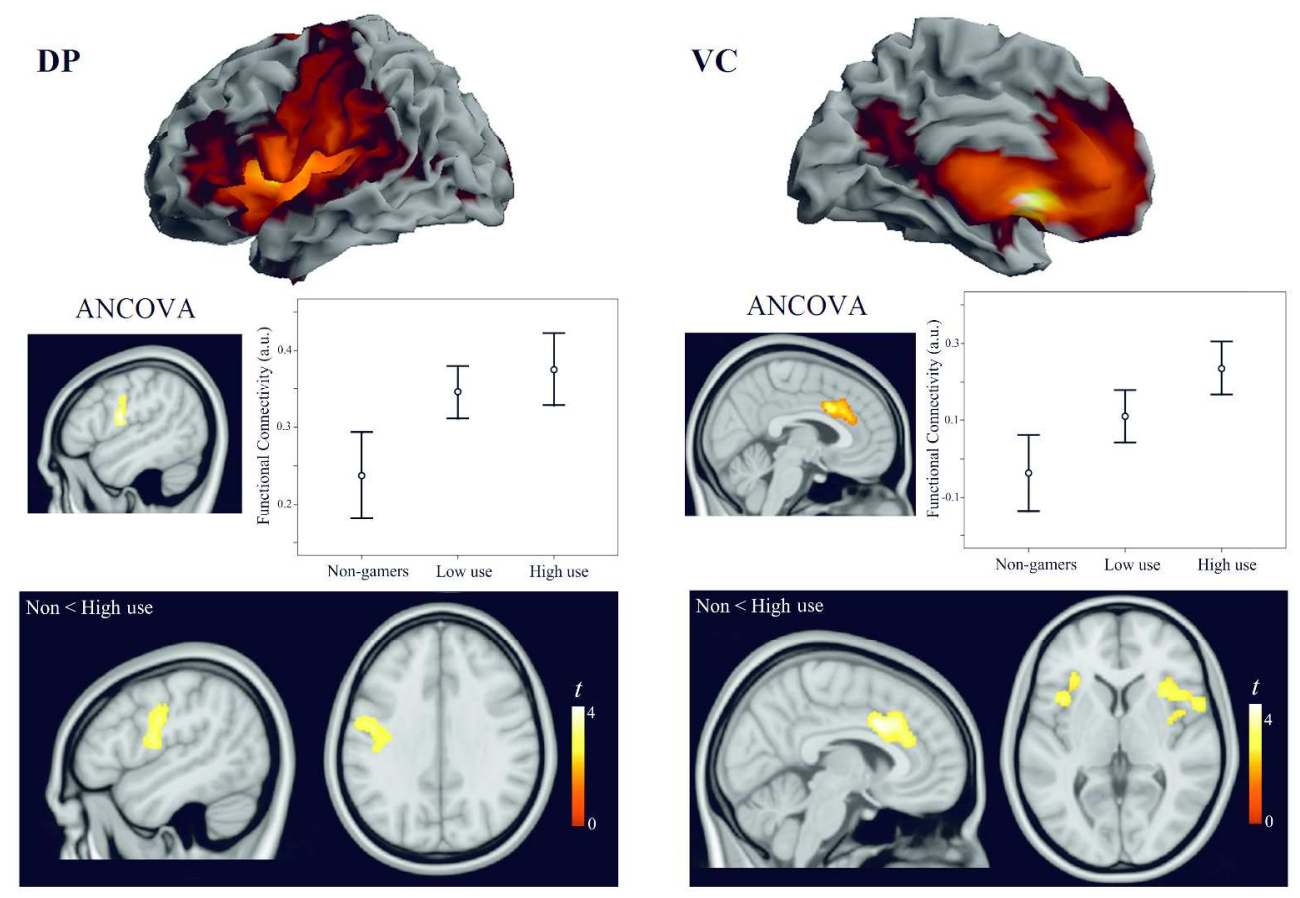

Figure 4. Gaming group differences in basal ganglia functional connectivity. ANCOVA indicated significant changes in functional connectivity between the dorsal putamen (DP) and motor cortex at the level of the frontal operculum. In the ventral caudate (VC) analysis, the most relevant finding involved the anterior cingulate cortex. Mean and $95 \%$ CIs are plotted, reflecting region peak differences among gaming groups. The bottom left and right images illustrate corresponding differences identified between non-gamers versus high use gamers. The left hemisphere corresponds to the left side of axial views.

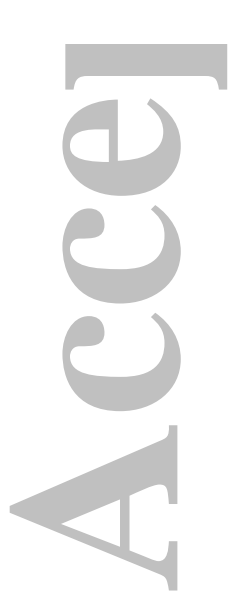

$279 \times 188 \mathrm{~mm}(300 \times 300$ DPI $)$ 


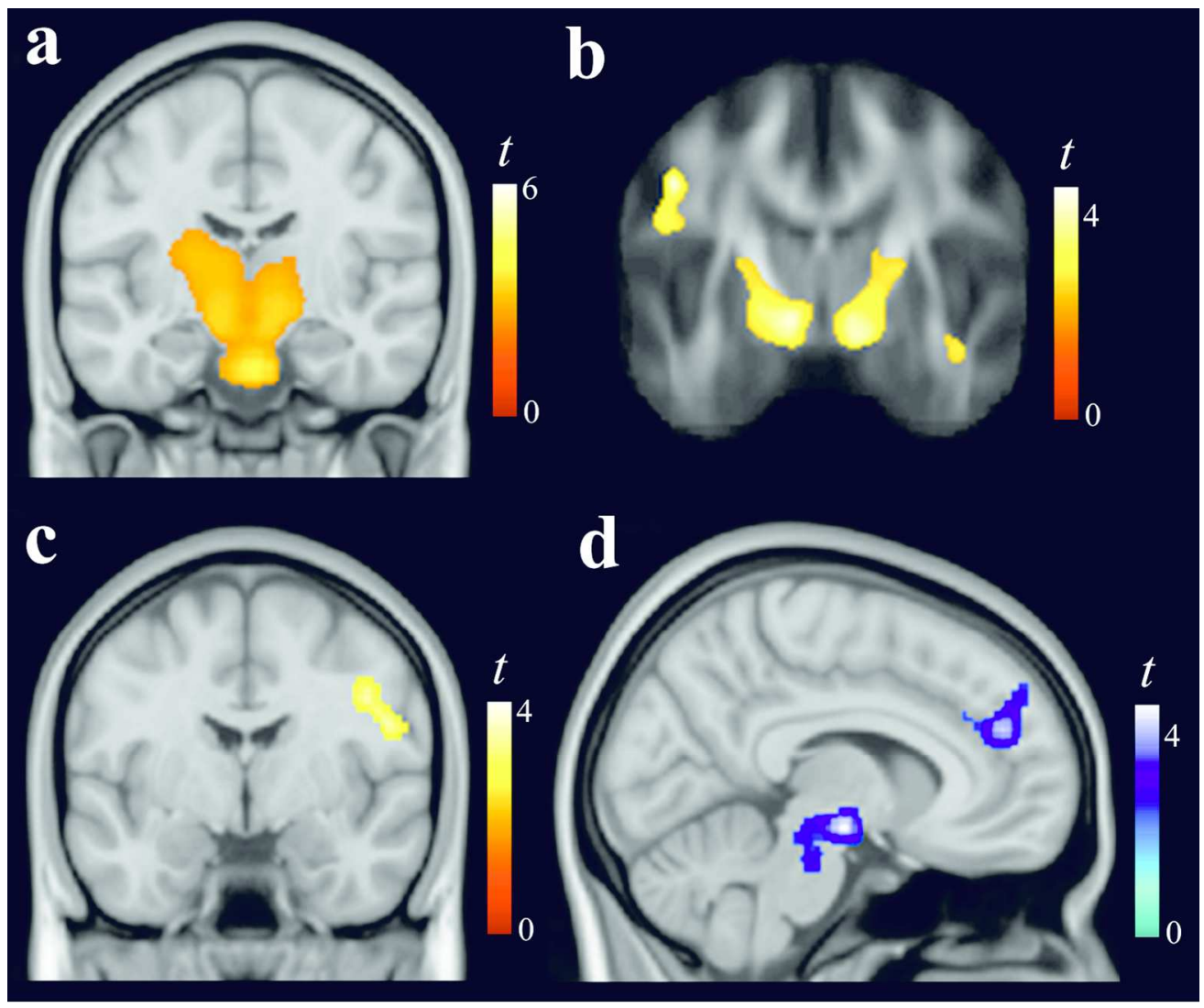

Figure 5. Correlations between age and brain measurements. White matter volume (a) increased significantly with age in projection tracts (and the visual system). Fractional anisotropy (b) increased with age mostly in the internal capsule and basal ganglia. Age was also associated with stronger functional connectivity between the putamen and motor cortex (c) and weaker functional connectivity between the caudate nucleus and the medial frontal cortex (d) and additionally with the upper brainstem (and amygdala).

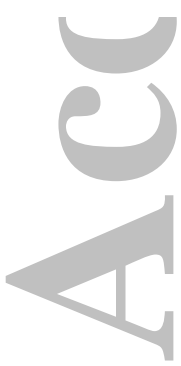

$132 \times 110 \mathrm{~mm}(300 \times 300 \mathrm{DPI})$ 


\section{University Library}

\section{- M M N E R VA A gateway to Melbourne's research publications}

Minerva Access is the Institutional Repository of The University of Melbourne

\section{Author/s:}

Pujol, J;Fenoll, R;Forns, J;Harrison, BJ;Martinez-Vilavella, G;Macia, D;Alvarez-Pedrerol, M;Blanco-Hinojo, L;Gonzalez-Ortiz, S;Deus, J;Sunyer, J

Title:

Video gaming in school children: How much is enough?

Date:

2016-09-01

\section{Citation:}

Pujol, J., Fenoll, R., Forns, J., Harrison, B. J., Martinez-Vilavella, G., Macia, D., AlvarezPedrerol, M., Blanco-Hinojo, L., Gonzalez-Ortiz, S., Deus, J. \& Sunyer, J. (2016). Video gaming in school children: How much is enough?. ANNALS OF NEUROLOGY, 80 (3), pp.424-433. https://doi.org/10.1002/ana.24745.

Persistent Link:

http://hdl.handle.net/11343/291638 